\title{
Review of oral oxymorphone in the management of pain
}

\author{
Paul Sloan \\ University of Kentucky \\ Medical Center, Department \\ of Anesthesiology, Lexington, \\ KY, USA
}

Correspondence: Paul Sloan University of Kentucky Medical Center, 800 Rose St., Suite N2 12, Lexington, KY 40536, USA

Tel +I 859323 5956, ext. 80103

$\mathrm{Fax}+\mathrm{I} 8593231080$

Cell 8595524403

Email paulsloan1956@yahoo.com

\begin{abstract}
Chronic cancer and nonmalignant pain (CNMP) is a common and major health problem afflicting approximately 40 million persons in the US. Most cancer patients, and many patients with CNMP, require opioid analgesics to obtain adequate pain relief. Oral oxymorphone is a new formulation of an existing parenteral opioid that has become available for the treatment of significant pain: acute postoperative, chronic arthritis, chronic low back, and chronic cancer pain. Oxymorphone is a typical mu-opioid agonist that is effective in both immediate- and extended-release (IR and ER) formulations. Oxymorphone is more lipid soluble than morphine, resulting in a rapid onset of action when given in tablet formulation, with a duration of action of approximately 4-6 hours in IR and 12 hours in ER preparations. Oxymorphone provides excellent pain relief for significant pain, with typical opioid side effects that are usually mild or moderate in intensity. Multiple double-blind, prospective, placebo-controlled clinical trials have demonstrated the clinical efficacy and safety of this new oral opioid preparation. Oral oxymorphone is an effective opioid that provides a new therapeutic option for the physician.

Keywords: chronic pain, oxymorphone, opioids, extended-release, sustained-release, cancer pain
\end{abstract}

\section{Introduction}

Chronic pain, including cancer pain and chronic nonmalignant pain (CNMP), is a common and major health problem afflicting a significant number of patients, resulting in personal suffering, reduced productivity, and substantial health care costs. Musculoskeletal conditions such as low back pain, osteoarthritis, and myofascial pain are the leading causes of disability in individuals of working age (Yelin and Callahan 1995), and recently the Centers for Disease Control and Prevention issued estimates of the number of adults with arthritis and chronic joint symptoms at 70 million persons (CDC 2002). Total disability expenditures among US working adults cost the country over US\$200 billion per year and social security insurance benefits are far outstripping the increase in the working population insured for disability (Sloan and Babul 2006). Untreated chronic pain has been documented to interfere with sleep patterns, increase anxiety and depression, decrease quality of life, interfere with social relationships, and decrease the ability of a patient to cope with life (McCarberg 2007). Clearly, the treatment of patients suffering from CNMP is required from a humanitarian viewpoint in addition to the practice of good medicine.

Treatment for CNMP is frequently, and appropriately, initiated with nonopioid analgesics such as acetaminophen, and nonsteroidal anti-inflammatory drugs (NSAIDs). Not only do all of these agents have their own set of adverse reactions (such as gastric ulcers, renal and liver toxicity, and bleeding) but many patients fail to obtain effective pain relief with analgesics that have a ceiling effect. Only in recent years have opioid analgesics been recommended and evaluated in controlled clinical trials for the relief of CNMP (Portenoy 1996; Markenson et al 2005). While opioids 
have been known to provide excellent analgesia since ancient times, a concern of both clinicians and the general public with the use of chronic opioids to treat CNMP was the risk of addiction. A current consensus viewpoint is that most patients with CNMP can obtain chronic pain relief with opioids at low opioid addiction risk (Gagnon et al 2007), particularly when addiction risk assessment tools are used during initiation of opioid therapy (Kalso et al 2004; Belgrade et al 2006). The careful use of chronic opioids in the treatment of CNMP is thus becoming more prevalent. Chronic opioids, traditionally used in adults alone, have recently been recommended in children with chronic pain from rheumatic arthritis refractory to standard analgesic therapies (Kimura and Walco 2007). This review paper will focus on oral oxymorphone extendedrelease (ER) in the management of CNMP.

Chronic pain is common among cancer patients in the end stages of life, is one of the most feared consequences of cancer, and is a major contributor to the morbidity associated with cancer (Sloan and Melzack 1999). It is well established that orally administered, chronic opioids titrated to pain relief among cancer patients with pain will provide effective analgesia for the majority of patients (Melzack et al 1976). While early clinical trials demonstrated the efficacy of immediaterelease (IR) oral opioids in the treatment of cancer pain, the introduction of oral ER opioids has been viewed as a major advance in the management of chronic cancer pain (Thirwell et al 1989). Oral opioids are noninvasive, convenient, easy to titrate, inexpensive, and because cancer pain requires around-the-clock treatment, ER formulations providing 12- or 24-hour dosing are preferable (Dhaliwal et al 1995). This review paper will focus on oral oxymorphone ER in the management of cancer pain.

Opioids have been used in the effective treatment of postoperative pain since the invention of the hypodermic syringe and hollow needle in the 1850s (Brownstein 1993). Acute postsurgical pain is both common and frequently undertreated (Apfelbaum et al 2003), and becoming increasingly associated with same-day outpatient surgery. With varying and unpredictable levels of patient pain there is a need for flexible analgesic modalities that can be adjusted quickly to the level of pain rating (Gimbel et al 2005). Oxymorphone IR may be particularly useful for the treatment of postsurgical pain and is a focus of this review paper. Although nonopioid methods of postoperative analgesia exist (eg, local anesthetic nerve blocks, local anesthetic wound infiltration systems, NSAIDs), standard intermittent oral opioid analgesics continue to be the mainstay for postoperative pain relief. It is likely that multimodal therapy using a combination of opioids, NSAIDs and other nerve blocks will achieve the best pain relief after surgery.

Oral oxymorphone has recently become available for the treatment of acute and chronic significant pain. Morphine and other opioids have been prescribed for many years in the treatment of cancer pain, and in the past decade for the treatment of CNMP, and have been found to be effective for the relief of moderate to severe pain (Sloan 2007). Why should a new oral opioid formulation, oxymorphone, be of interest to the current clinician? The answer lies in the observed variability in patient response to opioids in both analgesia and adverse events. Opioids are known to activate opioid receptors in the central nervous system (CNS); however, a given patient may or may not obtain adequate analgesia without intolerable side effects from a given opioid. There is, in other words, tremendous variability in an individual patient's analgesic response to a given opioid (Swegle and Logemann 2006). The reasons for this variable response to opioid analgesia include differences in drug potency, differences in drug metabolism, the rate of release of IR and ER formulations, differences in opioid receptor-binding affinity, concurrent medications, production of active opioid metabolites, and differences in balance of analgesic versus hyperalgesic action of opioids (Sloan et al 2005). Thus, in order to improve opioid analgesia in patients with chronic pain, clinicians will often rotate to a different opioid. In fact, $20 \%$ of cancer patients rotate through three or more opioids before achieving an acceptable balance between analgesia and side effects (Cherny et al 1995). Therefore, expanding the number of oral opioids available to our patients should help in terms of treatment options and dose flexibility. In addition, oxymorphone may hold specific chemical and pharmacokinetic properties (see below) that may be particularly favorable for use in chronic pain management.

\section{Chemistry}

Opioids have been used as analgesics since ancient times and were found in the early 1970 s to act upon opioid receptors in the central nervous system. Opioids are known to activate stereospecific G-protein opioid receptors on cell membranes but the exact mechanism of action is still not fully understood. In general, opioids act upon mu-, delta-, and kappareceptors on CNS neurons producing analgesia via decreased neuronal neurotransmitter release and decreased nociceptive impulse propagation (Kalso et al 2004). Opioids also may have seemingly paradoxical effects producing hyperalgesia (Wilder-Smith and Arendt-Nielsen 2006), although this is a less frequent, and likely dose-related, response. 
The mechanisms behind this paradoxical opioid effect are beyond the scope of this article but may pertain to dose-related opioid excitatory effects at the opioid receptor (Sloan and Hamann 2006), or may result from opioid stimulation of CNS glial cells which then release cytokines that "oppose" the analgesic effect of the opioid (Watkins et al 2007).

Oxymorphone is a mu-opioid agonist analgesic that has been available in parenteral formulation since 1959. Indeed, some of the earliest studies of parenteral opioids for the treatment of cancer pain and postoperative pain were completed using oxymorphone (Beaver and Feise 1977; Beaver et al 1977). Oxymorphone hydrochloride is a semisynthetic opioid agonist that modulates pain and provides significant analgesia because of specificity at the mu-opioid receptor (Metzger et al 2001). Its systematic name is 14-hydroxydihydromorphinone and it is synthesized (Figure 1) from thebaine or morphine, producing an odorless white crystal or powder, with the chemical formula $\mathrm{C}_{17} \mathrm{H}_{19} \mathrm{NO}_{4}$. Esterification of the hydroxyl groups will in fact produce a more potent opioid but none is currently available commercially.

Compared with other opioids, oxymorphone is more lipid soluble than morphine or oxycodone (Poyhia and Seppala 1994) which results in a more rapid transfer across the blood - brain barrier (Metzger et al 2001). Because of this rapid entry into the brain, the time to maximum plasma concentration $\left(\mathrm{T}_{\max }\right)$ of oxymorphone IR in humans is shorter ( $0.5 \mathrm{~h})$ compared with morphine IR (1.2 hours) or oxycodone IR (1.5 hours) (Adams and Ahdieh 2005). It is possible that the shorter $\mathrm{T}_{\max }$ will result in more rapid onset of analgesia compared with other opioids.

Oxymorphone is available for parenteral injection (Numorphan ${ }^{\circledR}: 1$ and $1.5 \mathrm{mg} / \mathrm{mL}$ ), suppository (Numorphan ${ }^{\circledR}$ : $5 \mathrm{mg}$ ), oral IR tablets (Opana ${ }^{\circledR}: 5$ and $10 \mathrm{mg}$ ), and oral ER tablets (Opana ${ }^{\circledR}$ ER: 5, 10, 20, and $40 \mathrm{mg}$ ).

\section{Pharmacokinetics}

This review will only consider the pharmacology and efficacy of the newly released oral IR and ER tablet formulations of oxymorphone.

\section{Oxymorphone IR tablets}

Oxymorphone is well absorbed from the gut but the liver eliminates most of the drug during the first-pass; thus the oral bioavailability of oxymorphone is $10 \%$ (Sloan et al 2005). The astute reader will not interpret this as incompatible with good clinical efficacy, as oral morphine also has a low oral bioavailability of $15 \%-30 \%$ and is clearly effective in the management of chronic cancer pain (Thirwell et al 1989). The absorbed lipid-soluble oxymorphone easily and rapidly enters the CNS, binding to mu-opioid receptors to produce analgesia. Oxycodone, by contrast, exhibits a substantial analgesic effect from binding to kappa-opioid receptors in the spinal cord (Ross and Smith 1997) which may explain, in part, the individual variable analgesic and side effect response to a given opioid. The volume of distribution of oxymorphone is $3 \mathrm{~L} / \mathrm{kg}$ (similar to morphine) with protein binding of about $10 \%$.
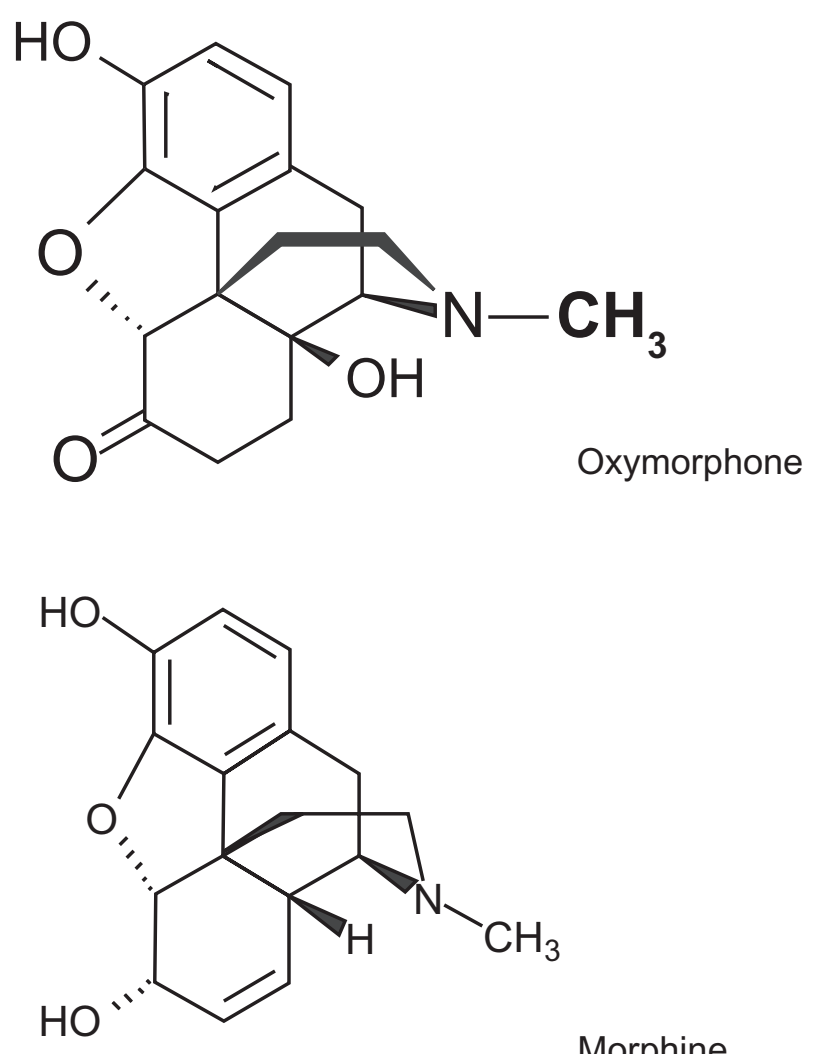

Morphine

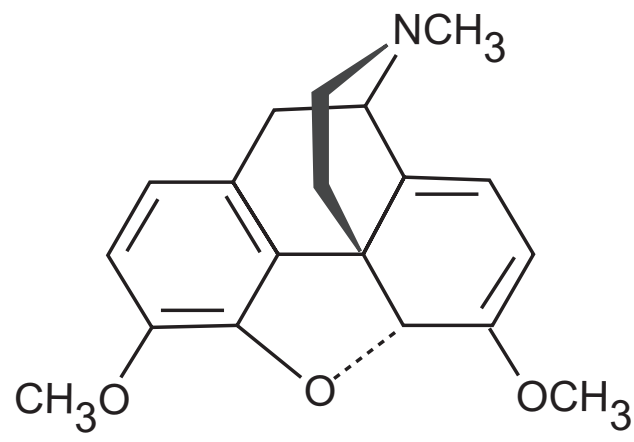

Thebaine

Figure I Oxymorphone: structure similar to morphine and thebaine. 
Plasma concentrations reach $\mathrm{T}_{\text {max }}$ at 0.5 hours after single doses $(5,10$, or $20 \mathrm{mg}$ ) in healthy volunteers (Adams and Ahdieh 2005). This correlates well with a recent clinical trial of oxymorphone IR $5 \mathrm{mg}$ tablets for the treatment of acute postsurgical pain where onset and time to peak analgesia was seen at 0.5 and 1.0 hours, respectively (Gimbel et al 2005). In a volunteer study, maximum plasma concentrations $\left(\mathrm{C}_{\max }\right)$ and area under the plasma concentration versus time curve (AUC) increased proportional to the single dose range of 5-20 mg, confirming linear pharmacokinetics. These simple, but important, data imply that an increase in dose in the clinical setting will produce predictable increases in oxymorphone plasma levels (Adams and Ahdieh 2005). After a 1-week dosing of oxymorphone every 6 hours, similar linear results (Figure 2) were observed for $\mathrm{C}_{\max }$ and $\mathrm{AUC}$
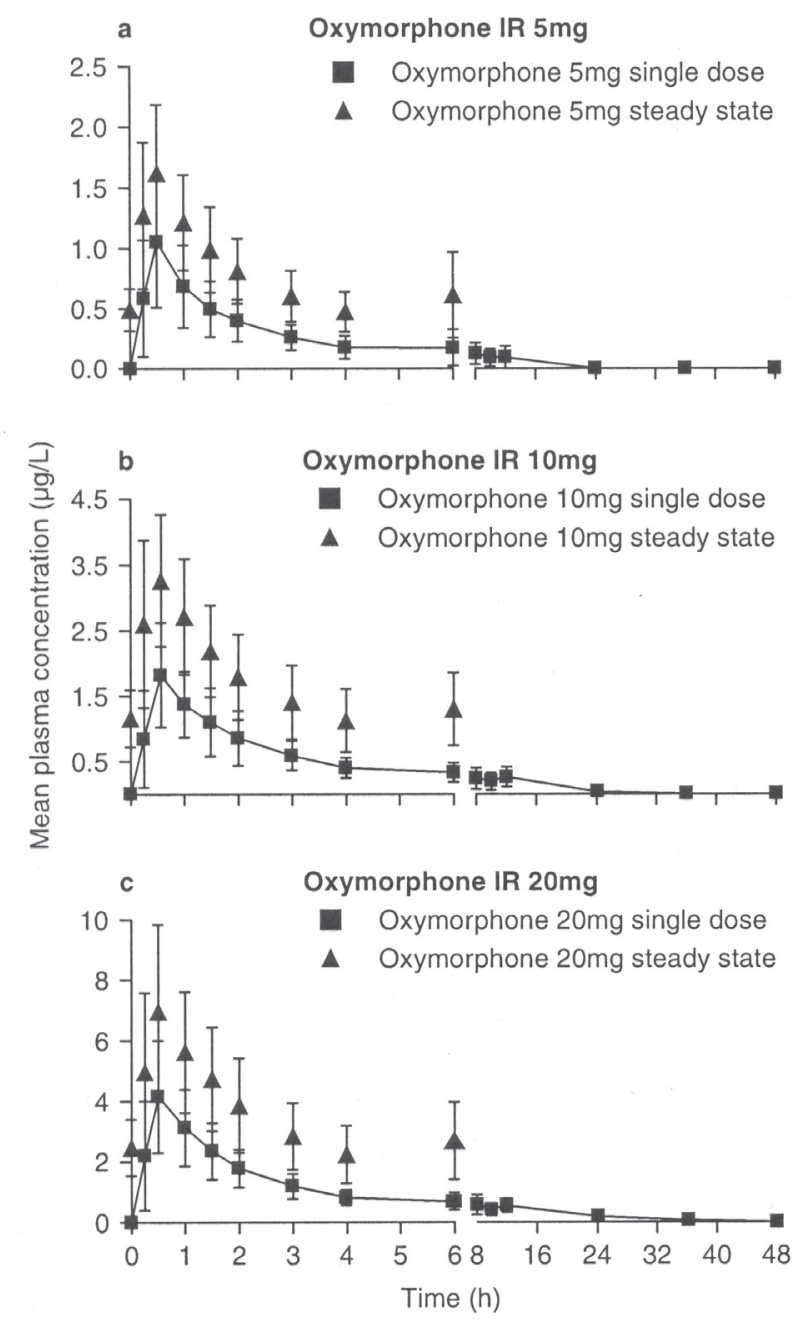

Figure 2 Mean single-dose and steady-state ( $q 6 \mathrm{~h}$ dosing) plasma concentrations of oxymorphone IR (5, I0, and $20 \mathrm{mg}$ doses) in healthy volunteers. Reprinted with permission from Adams MP, Ahdieh H.2005. Single- and multiple-dose pharmacokinetic and dose-proportionality study of oxymorphone immediate-release tablets. Drugs $R D$, 6:91-9. Copyright (C) 2005 Lippincott Williams and Wilkins. at steady state. Plasma oxymorphone concentrations taken during the multiple-dose period showed that $\mathrm{T}_{\max }$ remained at 0.5 hours and that steady-state was achieved after 3 days of oxymorphone IR dosing (Adams and Ahdieh 2005). Ingestion of food along with oxymorphone increases the $\mathrm{C}_{\max }$ by $38 \%-50 \%$ (The Medical Letter 2007); thus it may be advisable to take the drug on an empty stomach.

Oxymorphone is highly metabolized in the liver (principally conjugation with glucuronide) with only $2 \%$ excreted unchanged by the kidney (Adams and Ahdieh 2005). The main metabolite is oxymorphone-3-glucuronide, with a lesser amount of 6-OH-oxymorphone formed. The analgesic activity of oxymorphone-3-glucuronide is unknown. At steady-state conditions, 6-OH-oxymorphone plasma concentrations approximate those of oxymorphone, and the glucuronide metabolite is 90 -fold higher than oxymorphone (Endo Pharmaceuticals 2007). During dose escalation, the oxymorphone metabolites also increase in a proportional manner. With healthy volunteer studies (Adams and Ahdieh 2005) the elimination half-life was approximately 8 hours, about double that of both morphine and oxycodone.

\section{Oxymorphone ER tablets}

Oxymorphone ER is a new tablet formulation of oxymorphone that utilizes the proprietary TIMERx ${ }^{\circledR}$ technology (Penwest Pharmaceuticals) which allows 12-hour dosing of medication (Oxymorphone-Endo/Penwest 2003). This controlled-release technology inserts the opioid into an agglomerated hydrophilic matrix which releases the drug (as water penetrates the matrix) to sustain plasma levels during the 12-hour dosing interval.

As with oxymorphone IR, volunteer studies on healthy persons (Adams and Ahdieh 2004) have characterized the basic pharmacokinetics of oxymorphone ER. Singledose pharmacokinetics demonstrate dose-proportionality and linearity over the dose range of 5-40 $\mathrm{mg}$. Oxymorphone metabolites also increase in a linear fashion after single- and multiple-dose administration. The elimination half-life from single-dosing of oxymorphone ER was approximately 10 hours, quite similar to the half-life of oxymorphone IR. Figure 3 demonstrates the plasma levels of oxymorphone sustained over the 12-hour dosing interval and with very little fluctuation in plasma concentration over the dosing interval. Steady-state conditions were achieved after 3 days of 12-hour dosing. $T_{\max }$ occurred at just 1.5-3.5 hours; however a careful inspection of Figure 3 reveals that $\mathrm{T}_{\max }$ was sustained until approximately 6 hours, 


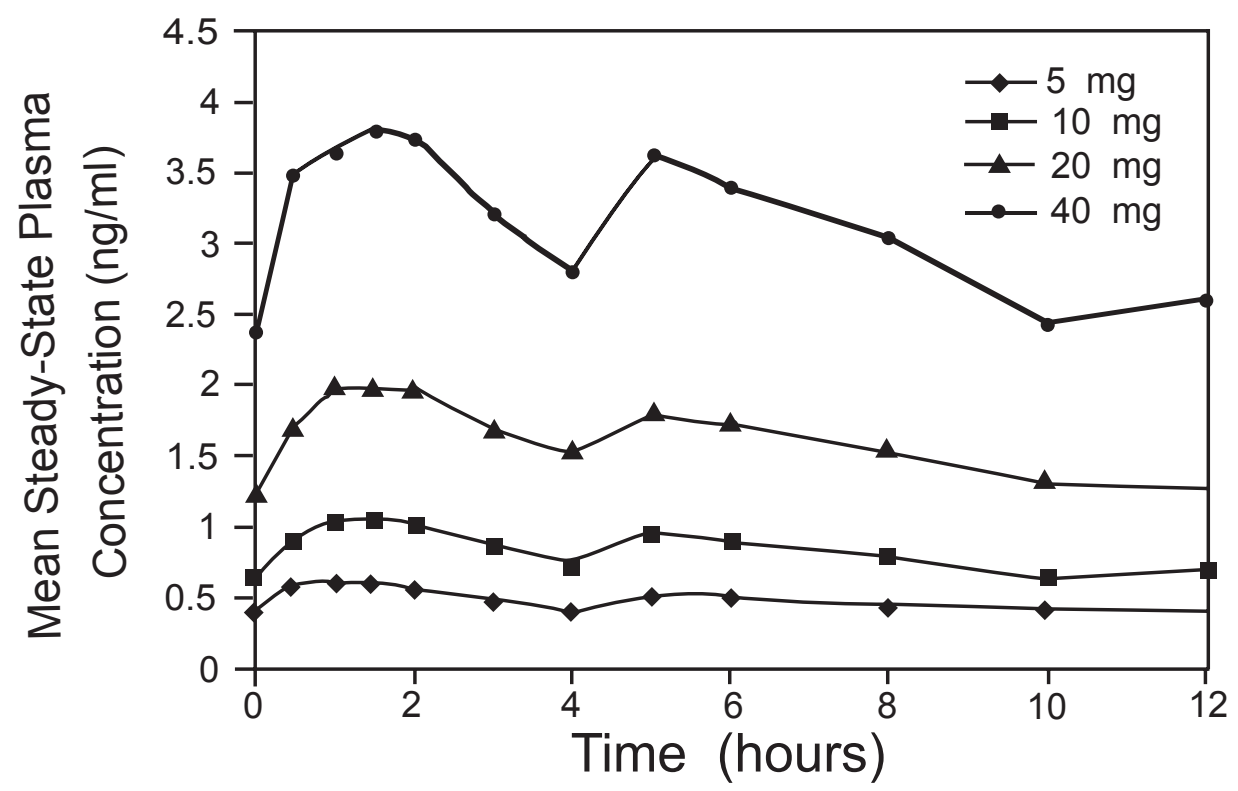

Figure 3 Mean steady-state plasma concentrations of oxymorphone ER over the 12-hour dosing interval in healthy volunteers. Reprinted with permission from Adams MP, Ahdieh H. 2004. Pharmacokinetics and dose-proportionality of oxymorphone ER and its metabolites: results of a randomized crossover study. Pharmacotherapy, 24:468-76. Copyright @ 2004 Pharmacotherapy Publications.

at which time the plasma concentration started to fall to trough levels. These pharmacokinetic data correlate quite well with the single published clinical study of oxymorphone ER for the treatment of postsurgical pain, in which analgesia was noted to occur approximately 2 hours after oxymorphone administration, and lasted at least 12 hours (Ahdieh et al 2004).

Oxymorphone from the ER preparation is obviously metabolized by the liver in the same fashion as for the IR compound. As doses increase, plasma levels of oxymorphone metabolites (6-hydroxymorphone and oxymorphone-3-glucuronide) increase in a linear fashion after both single- and multiple-dose administration (Ahdieh et al 2004).

In vitro studies show no effect of alcohol on the release of oxymorphone ER from the matrix; however, a volunteer study (Endo Pharmaceuticals 2007) showed that following the concomitant administration of $240 \mathrm{~mL}$ of $20 \%$ ethanol, the $\mathrm{C}_{\max }$ increased on average by $31 \%$, with a wide range of variation. Thus, as with all opioids, consumption of alcohol beverages should be avoided. A volunteer study in healthy individuals showed that when oxymorphone was taken with a high-fat meal, the $\mathrm{C}_{\max }$ was increased by $38 \%$ compared with fasted volunteers (Endo Pharmaceuticals 2007). It is recommended to take oxymorphone on an empty stomach. As with many medications, plasma levels of oxymorphone were found to be higher (40\%) in elderly than younger subjects.
There is no known gender effect on the pharmacokinetics of oxymorphone.

\section{Clinical application Oxymorphone IR tablets}

Oxymorphone IR is recommended for the treatment of moderate to severe pain, typically for the management of postsurgical pain when nonopioid analgesics are not expected to provide adequate analgesia. Opioid IR medications are also used as PRN rescue analgesics for patients on long-acting opioids for the management of cancer or CNMP. Traditionally, short-acting opioids have been used in initial trials for patients with CNMP to determine the correct daily dose of opioid, followed by conversion to the corresponding ER formulation of the same opioid for long-term use (Sinatra 2006). Some clinicians continue to utilize IR opioids long term for the treatment of CNMP as the incidence of opioid side effects is often similar between IR and ER opioid preparations (Hale et al 1999).

Two recent clinical trials have assessed the efficacy and safety of oral oxymorphone IR for the treatment of postoperative pain. Gimbel and Ahdieh reported results from a multicenter, double-blind, randomized, prospective and placebo-controlled clinical trial in 258 patients receiving total hip or total knee replacement surgery (Gimbel and Ahdieh 2004). Patients were randomized to receive in the postoperative period a single dose of oxymorphone IR (10, 
20 , or $30 \mathrm{mg}$ ), oxycodone $10 \mathrm{mg}$, or placebo within 6 hours of discontinuing parenteral opioids and when pain intensity was rated at $>45 \mathrm{~mm}$ on a $0-100 \mathrm{~mm}$ pain visual analog scale (VAS). Patients were monitored for efficacy and side effects during an 8-hour period. Patients $(\mathrm{n}=164)$ who completed this single-dose phase entered a multiple-dose period and received either oxymorphone IR or oxycodone IR every 4-6 hours PRN for an additional 48 hours. All doses of oxymorphone IR provided greater postsurgical analgesia compared with placebo $(\mathrm{p}<0.05)$. The median time to "meaningful" pain relief for patients receiving oxymorphone IR was 1 hour. All oxymorphone IR groups maintained analgesia during the 48-hour multi-dose phase with median PRN dosing intervals of 7-9 hours. Of note, an analgesic plateau seemed to appear with the $20 \mathrm{mg}$ dose providing analgesia equal to the $30 \mathrm{mg}$ dose. Opioid-related side effects were typical (nausea, vomiting, somnolence), rated as mild - moderate, and similar between the two opioids. The same research group studied the efficacy and safety of a lower dose $(5 \mathrm{mg}$ ) of oxymorphone IR for the treatment of mild to moderate pain after outpatient knee arthroscopy (Gimbel et al 2005). This prospective, randomized, doubleblind, placebo-controlled trial studied 122 patients who took study medication on a PRN basis (maximum dose medication q1 h) and were followed for 8 hours postdose. Oxymorphone $5 \mathrm{mg}$ IR was effectively titrated by patients to relieve mild to moderate postoperative pain (pain VAS of approximately $35 / 100$ over 8 hours) compared with the placebo group $(\mathrm{p}<0.05)$ and the mean dosing interval was 1.9 hours. Side effects were common (approximately $50 \%$ ), mild (nausea, headache), and equal compared with the placebo group.

Oxymorphone IR was used open-label to determine daily dosing required in a study of oxymorphone ER; however, data on the oxymorphone IR phase were not published (Gabrail et al 2004). Two studies on the efficacy of oxymorphone ER for chronic pain (Sloan et al 2005; Katz et al 2007) have used oxymorphone IR as PRN rescue analgesics but have not published data specifically on the IR preparation.

\section{Oxymorphone ER tablets}

Oxymorphone ER has been studied most extensively as a 12-hour medication in the long-term treatment of chronic pain, with 2 published studies for cancer pain and 6 published studies for CNMP, for a total of 887 patients completing the various clinical trials. Other competing oral ER opioids in the US include morphine, oxycodone, and tramadol. Methadone, a long-acting oral opioid with a half-life of 1 day, is also used in the treatment of chronic pain, as is a long-acting transdermal preparation of fentanyl. Additional oral ER opioids under investigation or available in other countries include codeine, hydromorphone, hydrocodone, and dihydrocodeine. ER oral opioids were developed in the 1980s to improve quality of life for our patients by offering: improved convenience, improved compliance, improved pain control, reduced opioid side effects, reduced pill burden, reduced night time pain, and improved sleep (Sloan and Babul 2006). Clinical trials comparing comparable ER with IR opioids, however, often show little difference with quality of pain relief or frequency of side effects (Hays et al 1994; Stambaugh et al 2001). Nonetheless, ER products have reduced pill burden, improved convenience, and patients often prefer the ER formulation over an IR formulation in the setting of chronic pain treatment (Broomhead et al 1997).

Two clinical trials have been published demonstrating the effectiveness of oxymorphone ER for the treatment of chronic cancer pain. Sloan and colleagues assessed the efficacy and safety of oxymorphone ER compared to morphine ER or oxycodone ER, among 86 patients with cancer pain (Sloan et al 2005). Patients were titrated (open-label, prospective) and treated for 7 days on either morphine ER or oxycodone ER, and then crossed over for an additional 7 days of treatment at an estimated equianalgesic dose of oxymorphone ER. There were no significant differences in daily pain scores (Figure 4) between oxymorphone ER and the alternative opioids, indicating similar analgesia among groups. When compared with morphine ER, patients on oxymorphone ER used less rescue analgesics, suggesting that oxymorphone ER may have a longer duration of activity than morphine ER. At study conclusion, equianalgesic dose ratios of morphine ER and oxycodone ER (Figure 5) were calculated to be $1.8: 1$ and 1.2:1, respectively. The side effects were mild, typical for opioids (nausea, drowsiness), and similar for all opioids. Gabrail and colleagues (Gabrail et al 2004) reported results of a double-blind, crossover study of oxymorphone ER for the treatment of cancer pain. Forty patients completed the entire study which used IR oxymorphone or oxycodone to achieve adequate pain relief among patients, and then convert patients to the ER formulation for another 7-10 days. Patients were then crossed over (double-blind fashion) to the alternative medication for an additional 7-10-day period. Both oxymorphone ER and oxycodone ER provided excellent and comparable analgesia with low requirements for rescue analgesics. The equianalgesic ratio of oxycodone ER to oxymorphone ER was found to be 2:1. 


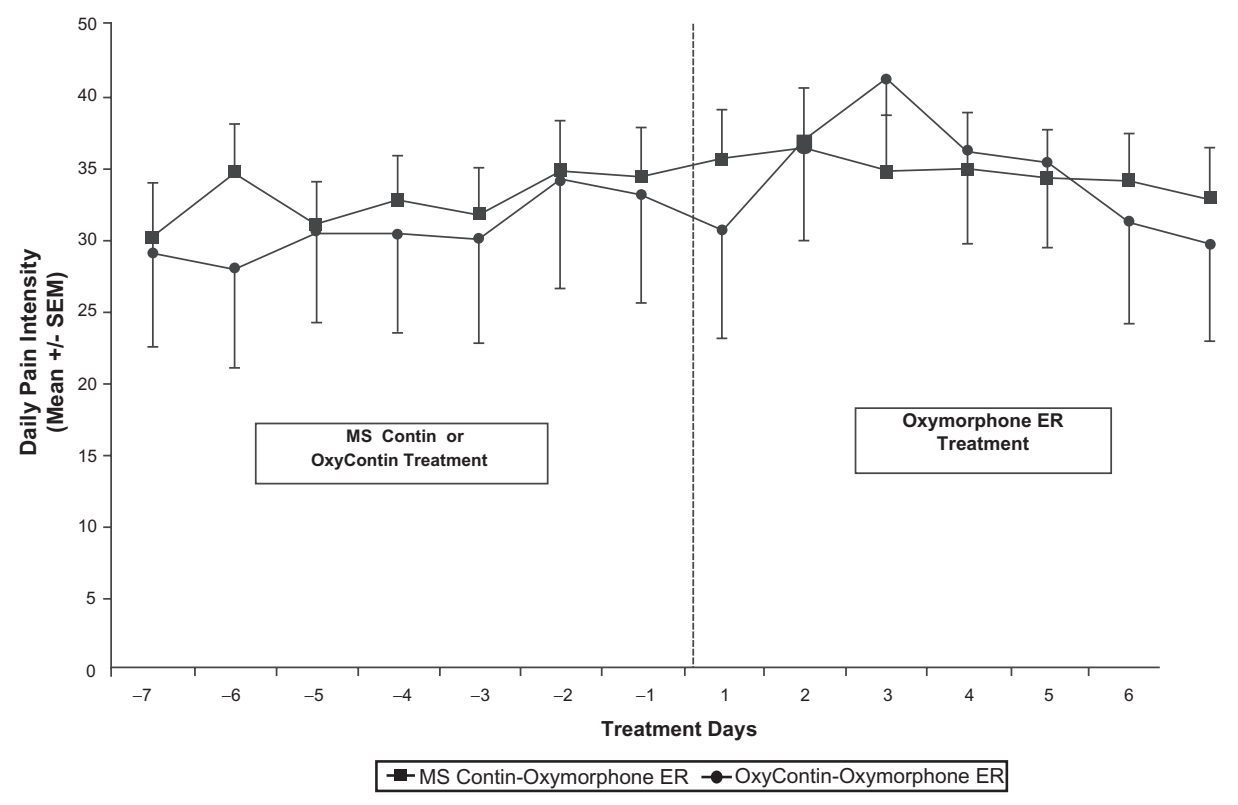

Figure 4 Mean (SEM) visual analog scale daily pain scores $\left(0=\right.$ no pain, $10=$ worst pain) for all cancer patients receiving MS Contin ${ }^{\circledast}\left(\right.$ morphine sulfate), OxyContin ${ }^{\circledast}($ oxycodone continuous release), and oxymorphone ER.

Six clinical trials have been published demonstrating the effectiveness of oxymorphone ER for the treatment of CNMP. The largest published clinical trial (Matsumoto et al 2005) found oxymorphone ER to be effective in the treatment of moderate-to-severe pain from osteoarthritis. Patients $(n=269)$ with osteoarthritis of the knee or hip completed the 1 month double-blind clinical trial comparing two doses of oxymorphone ER ( 20 and $40 \mathrm{mg} \mathrm{q} 12 \mathrm{~h}$ ) with oxycodone ER ( $20 \mathrm{mg} \mathrm{q} 12 \mathrm{~h}$ ) or placebo. Both doses of oxymorphone ER provided superior pain relief and improved physical functioning compared with the placebo group, with typical opioid side effects reported for both opioid medications. The longest prospective trial of oxymorphone ER (McIlwain and Ahdieh 2005) was completed in patients with pain from osteoarthritis who were followed over a 1 -year period. This open-label extension study followed 153 patients (with 61 patients completing the 1-year trial) and achieving pain relief with titration of oxymorphone 20 or $40 \mathrm{mg}$ twice daily. No rescue opioid analgesics were used during the study. Pain relief was excellent (Figure 6) with a mean current VAS pain intensity score at end-of-study of $20 \mathrm{~mm}$, an approximate $60 \%$ reduction in pain rating compared with initiation of the trial. The mean daily dose of oxymorphone ER at end-of-study was $62 \mathrm{mg}$, and remained very stable throughout the 1-year trial. Typical opioid side effects were reported and were the most common reason for withdrawal from study. No serious adverse events were noted. Interestingly, no instances of opioid addiction or drug misuse were recorded.

Two very recent studies have reported the use of oxymorphone ER for the relief of chronic low back pain (CLBP) over a 3-month clinical trial. Katz and colleagues (Katz et al 2007) titrated 325 opioid-naïve patients with CLBP using oxymorphone ER given twice daily, and then randomized patients to either continue the oxymorphone ER or receive placebo. Oxymorphone IR was always available for rescue analgesia. Two hundred and five patients were stabilized on oxymorphone ER with a mean VAS pain intensity rating decrease from 69 at screening to 23 at completion of titration. After randomization, and as expected, pain intensity increased significantly more in the placebo group, in spite of available oxymorphone IR rescue opioid, compared with the opioid treatment group. The authors concluded that stabilized doses of oxymorphone ER were generally safe and effective over a 12-week double-blind treatment period in opioid-naïve patients with CLBP (Katz et al 2007). This study is important because patients with CLBP represent the largest group of patient visits to most chronic pain clinics. A similar clinical trial (Hale et al 2007) converted 143 patients with CLBP from their chronic opioid to oxymorphone ER, and then randomized the patients to either continue the oxymorphone ER or receive placebo. Pain intensity was better in the oxymorphone ER group compared with the placebo (with oxymorphone IR rescue analgesics) group, 


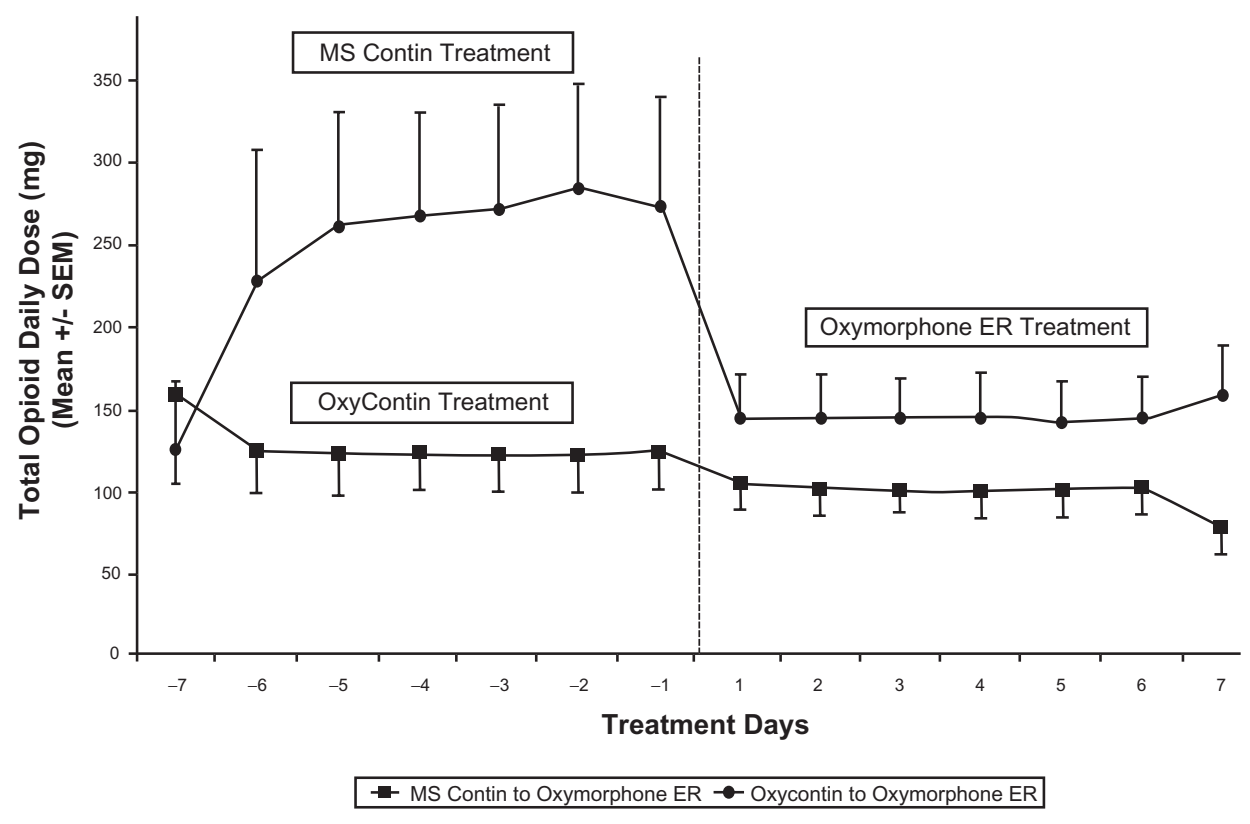

Figure 5 Mean (SEM) opioid daily dose for all cancer patients receiving MS Contin ${ }^{\circledast}$ (morphine sulfate), OxyContin ${ }^{\circledast}$ (oxycodone continuous release), and oxymorphone ER.

and opioid-related side effects were mild and similar between groups. For opioid-experienced patients with CLBP, oxymorphone ER provided long-term analgesia that was generally well tolerated (Hale et al 2007).

\section{Adverse effects}

Typical opioid side effects (nausea, vomiting, constipation, sedation, dry mouth) have been reported with all the published clinical trials to date, usually mild, with no serious adverse events yet reported. As with all opioids for chronic pain management, some patients will discontinue analgesics because of intolerable side effects. In published studies of oxymorphone to date, the highest rate of medication withdrawal secondary to side effects occurred among opioid-naïve patients started on a relatively high dose of oxymorphone ER (20 mg twice daily) with no titration period (McIlwain and Ahdieh 2005). It is likely that the rate of acceptance of oxymorphone as analgesic can be improved using a lower opioid dose (Gimbel et al 2005) with slow upward titration as is recommended for all opioids (Sloan and Babul 2006). Although no evidence of opioid misuse has been reported in available clinical trials, it is likely that oxymorphone ER has an opioid addiction potential similar to current available opioids.

Respiratory depression may occur with higher doses (The Medical Letter 2007), as with all opioids. Chronic oxymorphone use will result in physical dependence, which means that the drug should be tapered slowly rather than abrupt discontinuation. Physical dependence does not imply psychological addiction. Opioids have recently been implicated to interfere with the immune system (Sacerdote 2006). The interaction of oxymorphone with the immune system has not been investigated. Oxymorphone administered in large doses to rodents has not been found to be carcinogenic (Shuey et al 2007). Recent research has suggested that chronic opioids may reduce androgen levels in men by suppressing the hypothalamic-pituitary-gonadal axis (Daniel et al 2006). It is likely that oxymorphone may produce the same side effect of reduction in testosterone levels.

\section{Dosing guidelines}

Oxymorphone IR may be used as needed in the treatment of acute postsurgical pain with a dose of $5-10 \mathrm{mg}$ every 4 hours. The IR formulation will also be used as a breakthrough medication for patients on chronic oxymorphone ER for chronic pain management, given as a dose of $10 \%$ of the total daily oxymorphone ER dose every 2 hours as needed. Finally, for opioid-naïve patients, oxymorphone IR (5-10 mg q4-6 h) is often used to titrate patients to acceptable pain relief with slow upward dose titration and then convert patients to the ER formulation after arriving at a stable daily dose. Oxymorphone should be administered on an empty stomach. As with any opioid, it is vital to adjust the dosing regimen for each patient individually, taking into account the patient's prior analgesic therapy (Endo Pharmaceuticals 2007). 


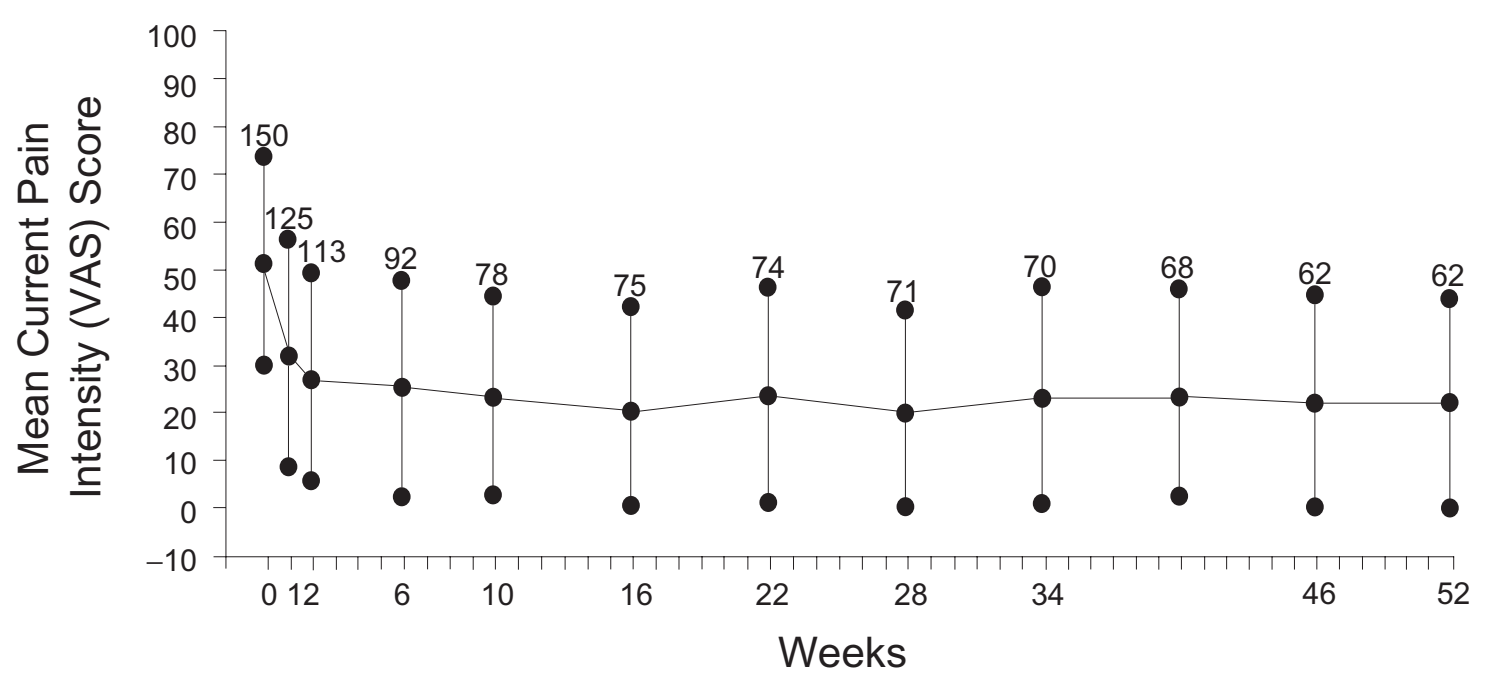

Figure 6 Mean current visual analog scale pain intensity $(0=$ no pain; $100=$ worst pain) over a one-year clinical trial of oxymorphone ER for arthritis pain. (The number of patients included in scoring is given above the error bar for each assessment) Reprinted with permission from Mcllwain H,Ahdieh H. 2005. Safety, tolerability, and effectiveness of oxymorphone extended-release for moderate to severe osteoarthritis pain: a one-year study. Am JTher, 12:106-12. Copyright (C) Lippincott Williams and Wilkins.

Because of individual patient variability in response to opioid analgesics, opioid rotation is becoming more common to achieve improved balance between opioid analgesic effect and adverse effects (Mercadante and Bruera 2006; Freye et al 2007). For opioid conversion to oxymorphone ER, oxycodone ER and morphine ER may be calculated to be equipotent and half as potent as oxymorphone ER, respectively (Sloan et al 2005). Because other opioids have not been directly compared with oxymorphone, published dose conversion tables may be used to convert other opioids first to morphine daily equivalents, and then complete the estimated conversion to oxymorphone. The clinician must always start the oxymorphone ER using approximately half of the calculated dose, since incomplete opioid cross tolerance exists. Once therapy has been initiated, pain relief and side effects should be closely monitored with dosage adjustment accordingly.

Oxymorphone ER should be prescribed every 12 hours. While other ER opioid formulations also recommend 12 hourly dosing, recent studies suggest that patients often use the medication on an 8-hourly schedule (Sinatra 2006). Because plasma levels of oxymorphone ER and analgesia are clearly sustained over 12 hours (Ahdieh et al 2004), it may truly be utilized as a 12-hour opioid formulation. Additional long-term studies are needed to determine if this is a legitimate advantage of oxymorphone ER over other opioid ER products.

The occasional cancer patient may use parenteral oxymorphone on a chronic daily basis. Given the low oral bioavailability of oxymorphone, parenteral oxymorphone may be converted to oral oxymorphone by a factor of 10 .

\section{Precautions}

Oxymorphone should be used cautiously (start with a low dose and titrate upward very slowly) in patients with hepatic disease as the plasma concentrations achieved are greater than in patients with normal liver function (Endo Pharmaceuticals 2007). The package insert advises that oxymorphone is contraindicated in patients with moderate or severe liver dysfunction (Endo Pharmaceuticals 2007). Oxymorphone bioavailability is reported to increase $57 \%-65 \%$ in patients with moderate to severe renal impairment (Endo Pharmaceuticals 2007), thus is should be administered cautiously and in reduced dosages. Oxymorphone has been found to be removed by hemodialysis (Lee et al 2005), yet the clinician would be wise to dose cautiously even for the patient on dialysis, since opioid accumulation may still occur (Foral et al 2007).

Oxymorphone should be used cautiously when combined with other CNS depressants, and in patients with severe pulmonary impairment. Oxymorphone should not be used in combination with alcohol products. Oxymorphone metabolism occurs in the liver, but does not significantly involve the cytochrome P450 enzyme system (Adams et al 2005), thus it differs from other opioids (such as oxycodone, methadone) and may reduce the potential for drug - drug interactions (Kivitz et al 2006).

\section{Conclusions}

The treatment of chronic cancer and nonmalignant pain requires the use of chronic opioids for many patients. While there are several opioids currently are on the market, new 
formulations are welcomed because the variable patient response to a particular opioid product results in many patients rotating to several different opioids before adequate balance of analgesia and side effect is achieved. Oral oxymorphone, in both IR and ER formulations, has become available for the treatment of acute and chronic significant pain. Clinical trials of oxymorphone demonstrate efficacy and safety, with typical opioid side effects, for the management of postsurgical pain, chronic arthritis pain, chronic low back pain, and chronic cancer pain. Oxymorphone has an advantage over some opioids in that it is now available in both parenteral and oral (IR and ER) formulations which leads to easy titration and conversion when patients experience formulation changes. Oxymorphone ER has been shown to be efficacious over the 12-hour dosing interval in clinical trials varying from 2 to 52 weeks. With proper dose titration, oral oxymorphone is an effective opioid that provides a new therapeutic option for the clinician. Future studies should continue to document the clinical efficacy of oral oxymorphone in long-term clinical trials (greater than 6 months) of cancer and CNMP, and should investigate the dose conversion from oxymorphone to other common opioids in use (eg, methadone, fentanyl patch).

\section{Disclosures}

Dr. Sloan has no current financial relationship or interest with any organization relevant to this review paper.

\section{References}

Adams MP, Ahdieh H. 2004. Pharmacokinetics and dose-proportionality of oxymorphone extended release and its metabolites: results of a randomized crossover study. Pharmacotherapy, 24:468-76.

Adams MP, Ahdieh H. 2005. Single- and multiple-dose pharmacokinetic and dose-proportionality study of oxymorphone immediate-release tablets. Drugs R D, 6:91-9.

Adams M, Pieniaszek Jr JH, Gammaitoni AR, et al. 2005. Oxymorphone extended release does not affect CYP2C9 or CYP3A4 metabolic pathways. J Clin Pharm, 45:337-45.

Ahdieh H, Ma T, Babul N, et al. 2004. Efficacy of oxymorphone extended release in postsurgical pain: a randomized clinical trial in knee arthroplasty. J Clin Pharmacol, 44:767-76.

Apfelbaum JL, Chen C, Mehta SS, et al. 2003. Postoperative pain experience: results from a national survey suggest postoperative pain continues to be undermanaged. Anesth Analg, 97:534-40.

Beaver WT, Feise GA. 1977. A comparison of the analgesic effect of oxymorphone by rectal suppository and intramuscular injection in patients with postoperative pain. J Clin Pharmacol, 17:276-91.

Beaver WT, Wallenstein SL, Houde RW, et al. 1977. Comparisons of the analgesic effects of oral and intramuscular oxymorphone and of intramuscular oxymorphone and morphine in patients with cancer. J Clin Pharmacol, 17:186-98.

Belgrade MJ, Schamber CD, Lindgren BR. 2006. The DIRE score: predicting outcomes of opioid prescribing for chronic pain. J Pain, 7:671-81.

Broomhead A, Kerr R, Tester W, et al. 1997. Comparison of a once-a-day sustained-release morphine formulation with standard oral morphine treatment for cancer pain. J Pain Symptom Manage, 14:63-73.
Brownstein MJ. 1993. A brief history of opiates, opioid peptides, and opioid receptors. Proc Natl Acad Sci USA, 90:5391-93.

[CDC] Centers for Disease Control and Prevention. 2002. Prevalence of self-reported arthritis or chronic joint symptoms among adults - United States, 2001. MMWR Morb Mortal Wkly Rep, 51:948-50.

Cherny NJ, Chang V, Frager G, et al. 1995. Opioid pharmacotherapy in the management of cancer pain: a survey of strategies used by pain physicians for the selection of analgesic drugs and routes of administration. Cancer, 76:1283-93.

Daniell HW, Lentz R, Mazer NA. 2006. Open-label pilot study of testosterone patch therapy in men with opioid-induced androgen deficiency. J Pain, 7:200-10.

Dhaliwal H, Sloan PA, Arkinstall W, et al. 1995. Randomized evaluation of controlled-release codeine and placebo in chronic cancer pain. J Pain Symp Manage, 10:612-23.

Endo Pharmaceuticals. 2007. Opana (oxymorphone hydrochloride) tablets [online]. Accessed 1 June 2007. URL: http:// www.opana.com/pdfs/ opana_ir_pi.pdf.

Foral PA, Ineck JR, Nystrom KK. 2007. Oxycodone accumulation in a hemodialysis patient. South Med J, 100:212-4.

Freye E, Anderson-Hillemacher A, Ritzdorf I, et al. 2007. Opioid rotation from high-dose morphine to transdermal buprenorphine (Transtec) in chronic pain patients. Pain Pract, 7:123-9.

Gabrail NY, Dvergsten C, Ahdieh H. 2004. Establishing the dosage equivalency of oxymorphone extended-release and oxycodone controlled release in patients with cancer pain: a randomized controlled study. Curr Med Res Opin, 20:911-8.

Gagnon AM, Kahan M, Srivastava A. 2007. Opioid use and abuse: is there a problem? Clin J Pain, 23:661-2.

Gimbel J, Ahdieh H. 2004. The efficacy and safety of oral immediate-release oxymorphone for postsurgical pain. Anesth Analg, 99:1472-7.

Gimbel JS, Walker D, Ma T, et al. 2005. Efficacy and safety of oxymorphone immediate release for the treatment of mild to moderate pain after ambulatory orthopedic surgery: results of a randomized, double-blind, placebo-controlled trial. Arch Phys Med Rehabil, 86:2284-9.

Hale ME, Fleischmann R, Salzman R, et al. 1999. Efficacy and safety of controlled-release versus immediate-release oxycodone: randomized, double-blind evaluation in patients with chronic pain. Clin J Pain, 15:179-83.

Hale ME, Ahdieh H, Ma T, et al. 2007. Efficacy and safety of Opana ER (oxymorphone extended release) for relief of moderate to severe chronic low back pain in opioid-experienced patients: a 12-week, randomized, double-blind, placebo-controlled study. J Pain, 8:175-84.

Hays H, Hagen N, Thirlwell M, et al. 1994. Comparative clinical efficacy and safety of immediate and controlled release hydromorphone formulations in severe cancer pain. Cancer, 74:1808-16.

Kalso E, Edwards JE, Moore RA, et al. 2004. Opioids in chronic non-cancer pain: systematic review of efficacy and safety. Pain, 112:372-80.

Katz N, Rauck R, Ahdieh H, et al. 2007. A 12-week, randomized, placebocontrolled trial assessing the safety and efficacy of oxymorphone extended release for opioid-naive patients with chronic low back pain. Curr Med Res Opin, 23:117-28.

Kimura Y, Walco GA. 2007. Treatment of chronic pain in pediatric rheumatic disease. Nat Clin Pract Rheumatol, 3:210-8.

Kivitz A, Ma C, Ahdieh H, et al. 2006. A 2-week, multicenter, randomized, double-blind, placebo-controlled, dose-ranging, phase III trial comparing the efficacy of oxymorphone extended release and placebo in adults with pain associated with osteoarthritis of the hip or knee. Clin Ther, 28:352-64.

Lee MA, Leng MEF, Cooper RM. 2005. Measurements of plasma oxycodone, noroxycodone and oxymorphone levels in a patient with bilateral nephrectomy who is undergoing haemodialysis. Palliat Med, 19:259-60.

Markenson JA, Croft T, Zhang PG, et al. 2005. Treatment of persistent pain associated with osteoarthritis with controlled-release oxycodone tablets in a randomized controlled clinical trial. Clin J Pain, 21:524-35. 
Matsumoto AK, Babul N, Ahdieh H. 2005. Oxymorphone extended-release tablets relieve moderate to severe pain and improve physical function in osteoarthritis: results of a randomized, double-blind, placebo- and active-controlled phase III trial. Pain Med, 6:357-66.

McCarberg B. 2007. Tramadol extended-release in the management of chronic pain. Ther Clin Risk Manage, 3:1-10.

McIlwain H, Ahdieh H. 2005. Safety, tolerability, and effectiveness of oxymorphone extended-release for moderate to severe osteoarthritis pain: a one-year study. Am J Ther, 12:106-12.

Melzack R, Ofiesh JG, Mount BM. 1976. The Brompton mixture: effects on pain in cancer patients. Can Med Assoc J, 115:125-9.

Mercadante S, Bruera E. 2006. Opioid switching: a systematic and critical review. Cancer Treat Rev, 32:304-15.

Metzger TG, Paterlini MG, Ferguson DM, et al. 2001. Investigation of the selectivity of oxymorphone-and naltrexone-derived ligands via site-directed mutagenesis of opioid receptors: exploring the "address" recognition locus. J Med Chem, 44:857-62.

Oxymorphone-Endo/Penwest. 2003. Drugs, 4:204-6.

Penwest Pharmaceuticals Co. 2007. TIMERx technical description. Accessed 1 June 2007. URL: http://www.penwest.com/timerx_description.html.

Portenoy RK. 1996. Opioid therapy for chronic non-malignant pain: a review of the critical issues. J Pain Symptom Manage, 11:203-17.

Poyhia R, Seppala T. 1994. Liposolubility and protein binding of oxycodone in vitro. Pharmacol Toxicol, 74:23-7.

Ross FB, Smith MT. 1997. The intrinsic antinociceptive effects of oxycodone appear to be kappa-opioid receptor mediated. Pain, 73:151-7.

Sacerdote P. 2006. Opioids and the immune system. Palliat Med, 20:S9-15.

Shuey DL, Woodland C, Tremblay C, et al. 2007. Oxymorphone hydrochloride, a potent opioid analgesic, is not carcinogenic in rats or mice. Toxicol Sci, 96:162-73.

Sinatra R. 2006. Opioid analgesics in primary care: challenges and new advances in the management of noncancer pain. J Am Board Fam Med, 19:165-77.
Sloan PA. 2007. Neuraxial pain relief for intractable cancer pain. Curr Pain Headache Rep, 11:283-9.

Sloan PA, Babul N. 2006. Extended-release opioids for the management of chronic non-malignant pain. Exp Opin Drug Deliv, 3:489-97.

Sloan PA, Hamann SR. 2006. Ultra low-dose opioid antagonists to enhance opioid analgesia. J Opioid Manage, 2:295-304.

Sloan PA, Slatkin NE, Ahdieh H. 2005. Effectiveness and safety of oral extended-release oxymorphone for the treatment of cancer pain: a pilot study. Supp Care Cancer, 13:57-65.

Sloan PA, Melzack R. 1999. Long-term patterns of morphine dosage and pain intensity among cancer patients. Hosp J, 14:35-47.

Stambaugh JE, Reder RF, Stambaugh MD, et al. 2001. Double-blind, randomized comparison of the analgesic and pharmacokinetic profiles of controlled- and immediate-release oral oxycodone in cancer pain patients. J Clin Pharmacol, 41:500-6.

Swegle JM, Logemann C. 2006. Management of common opioid-induced adverse effects. Am Fam Physician, 74:1347-54.

The Medical Letter on Drugs and Therapeutics. 2007. Oral oxymorphone (Opana). The Med Letter, 49:3-4.

Thirlwell MP, Sloan PA, Maroun JA, et al. 1989. Pharmacokinetics and clinical efficacy of oral morphine solution and controlled-release morphine tablets in cancer patients. Cancer, 63:2275-83.

Watkins LR, Hutchinson MR, Ledeboer A, et al. 2007. Glia as the "bad guys": implications for improving clinical pain control and the clinical utility of opioids. Brain Behav Immun, 21:131-46.

Wilder-Smith OHG, Arendt-Nielsen L. 2006. Postoperative hyperalgesia. Anesthesiology, 104:601-7.

Yelin E, Callahan LF; for National Arthritis Data Work Groups. 1995. The economic cost and social and psychological impact of musculoskeletal conditions. Arthritis Rheum, 38:1351-62. 
